\title{
Optimal SF Allocation in LoRaWAN Considering Physical Capture and Imperfect Orthogonality
}

\author{
Christelle Caillouet*, Martin Heusse $^{\dagger}$, Franck Rousseau ${ }^{\dagger}$ \\ *Université Côte d'Azur, CNRS, I3S, Inria, France \\ †Univ. Grenoble Alpes, CNRS, Grenoble INP, LIG, F-38000 Grenoble France
}

\begin{abstract}
We propose a theoretical framework for maximizing the LoRaWAN capacity in terms of the number of end nodes, when they all have the same traffic generation process. The model optimally allocates the spreading factor to the nodes so that attenuation and collisions are optimized. We use an accurate propagation model considering Rayleigh channel, and we take into account physical capture and imperfect SF orthogonality while guaranteeing a given transmission success probability to each served node in the network. Numerical results show the effectiveness of our SF allocation policy. Our framework also quantifies the maximum capacity of single cell networks and the gain induced by multiplying the gateways on the covered area. We finally evaluate the impact of physical capture and imperfect SF orthogonality on the SF allocation and network performances.
\end{abstract}

\section{INTRODUCTION}

The growth of the Internet of Things (IoT) has opened up new challenges in recent years in the establishment of efficient networks, such as ensuring wireless communications while minimizing the energy consumption of sensors, and supporting an ever-increasing number of nodes connected to the Internet. Recently, Low Power Wide Area Networks, LPWANs, have drawn a lot of attention and experienced a significant growth. These radios are cheap and able to send and receive short messages over very long ranges, up to several kilometers, with very low power consumption, ensuring an autonomy of the sensors of more than 10 years [1].

LoRaWAN technology has recently established itself in the LPWAN market. It uses LoRa physical layer based on CSS, Chirp Spread Spectrum modulation, and a simple access method based on ALOHA. LoRaWAN networks have emerged as a promising solution for IoT in which several base stations are deployed over a large area to provide coverage and connectivity to sensors [2]. The end nodes communicate directly, in a single hop, with the gateways in a star network pattern.

The growing interest in this type of architecture poses several challenges. Indeed, the performance of a LoRaWAN network in terms of coverage, robustness, latency depends on the number of gateways deployed and the choice of parameters used for radio resources — bandwidth, spreading factor, coding, transmission power. The physical layer is based on CSS for robust communication in the sub-GHz ISM band and there are several spreading factors (SF) to choose from, which allows to trade data rate for range. The capacity of a cell is the number of nodes that a single gateway can handle before losses due to contention or attenuation reach unacceptable levels. These two factors are antagonistic, as more robust transmissions occupy the channel longer and thus increase the collision probability. Many papers address this question [1], [3], [4], [5], [6] to analytically determine the capacity of a LoRa cell.

In this work, we present a framework for optimally allocating the spreading factors (SF) in LoRaWANs, in order to maximize the number of served nodes. We consider a realistic propagation model with physical capture that may arise at the gateways, and express the potential interferers of each node considering intra-SF and inter-SF conditions for collisions [7].

Transmissions occurring with the same SF may collide if they happen simultaneously, except if one signal is significantly stronger than the other. The latter is called "capture effect", in which case the gateway can still decode the stronger transmission. The SFs are assumed to be quasi-orthogonal. The imperfect orthogonality of the SF has been studied and the SINR threshold for concurrent transmissions in different SFs have been quantified [8], [9], [10]. If two frames with different SF collide, both succeed if they are not significantly stronger than each other.

We develop a linear program to optimally allocate the spreading factors of the LoRaWAN nodes and guarantee a given reception success probability of the frames at the gateway, taking into account intra-SF and inter-SF collisions.

Optimal planning and management in LoRaWAN has drawn little attention until now. Cesana et al. optimally allocate the spreading factors, and estimate the data extraction rate for each spreading factor at the gateway [11]. They consider a log-distance path loss model without Rayleigh channel, and without capture effect. In comparison to this work, we consider the intra-SF and inter-SF collisions arising among spreading factors modeling the physical capture and imperfect SF orthogonality, to derive a model maximizing the number of served nodes while guaranteeing a given reception rate.

Zorbas et al. provide an analytical model to quantify the density of nodes a LoRaWAN gateway can serve [12]. They quantify the density of nodes for each spreading factor. However, their model computes an average probability in such a way that only spreading factors 7 and 8 are served, which is not realistic in practice since nodes located far away from the gateway are prevented to use high SFs and won't be served. They have recently extended this work to increase the network capacity using optimal SF allocation [13]. They use the same 
average transmission success probability among each SF to derive the SF boundaries in a LoRaWAN cell. They also limit their tests to a $500 \mathrm{~m}$ cell. In this work, we go further by extending the optimal SF allocation with several gateways in the network, while ensuring a given transmission success probability to each node.

Lim and Han [14] use stochastic geometry to compute de SF boundaries to maximize the packet success probability of the system. They derive a sub-optimal algorithm in a single cell LoRa network.

Section II presents the propagation model and how we consider physical capture and imperfect SF orthogonality, before defining the transmission success probability of each node. The optimal framework is described in Section III. Numerical results are presented in Section IV, showing the effectiveness of our SF allocation and quantifying the network capacity for different threshold of transmission success probability and number of gateways. Concluding remarks and further working directions are given in Section V.

\section{MODEL}

We consider a LoRaWAN with one or several gateways. To determine the allowed SFs (between SF7 and 12) for a node $i$ located at distance $d_{i}$ from a gateway, we define an accurate propagation model.

\section{A. Propagation model}

We assume that all nodes transmit at power $P_{t x}=14 \mathrm{dBm}$. The signal power at the gateway depends on the distance and Rayleigh fading. The transmission power is attenuated depending on the distance between the transmitter and the gateway:

$$
P_{r x}=P_{t x} * g\left(d_{i}\right)
$$

where $g\left(d_{i}\right)$ is the path-loss attenuation function based on the Okumura Hata model in a suburban environment with an antenna height of $15 \mathrm{~m}$. We consider a Rayleigh channel, so that the received signal power is affected by a random variable which follows an exponential distribution with unit mean.

In case there is no collision, a transmission is successfully received if the SNR at the receiver is above the minimum SNR for the corresponding spreading factor [8]. Therefore, the success probability of an isolated frame reception at distance $d_{i}$ is:

$$
H=e^{\left(-\frac{N q_{f}}{P_{r x}}\right)}
$$

where $N$ is the thermal noise for a $125 \mathrm{kHz}$-wide band: $N=$ $-174+N F+10 \log (B W)$, with $B W$ the signal bandwidth and $N F$ the receiver noise figure that can be considered to have a value of $6 \mathrm{~dB}$ in the hardware implementations, and $q_{f}$ is the minimum SNR for the corresponding spreading factor $f$. We assume an antenna gain of $6 \mathrm{~dB}$.

Instead of deterministically determining the possible SF that can be allocated to the nodes using the received power and the gateway sensitivity as in previous work [11], [12], we consider the property of the Rayleigh channel to estimate the success probability of frame reception. If this probability is above a given threshold $H \geq \beta$ for SF $f$, then we consider that the node located at distance $d_{i}$ can use the corresponding spreading factor. In case of several gateways, node $i$ can be allocated SF $f$ if this condition holds for at least one gateway.

\section{B. Physical capture}

LoRaWAN medium access control scheme can be well approximated by un-slotted ALOHA. Similarly to previous work [4], [11], we use the data extraction rate as the performance metric, defined as the ratio of received messages to transmitted messages over a period of time. For pure ALOHA systems it corresponds to:

$$
\operatorname{DER}=e^{\left(-2 N_{f} \cdot T^{f} \cdot \lambda\right)}
$$

where $N_{f}$ is the number of transmitters, $T^{f}$ is the packet airtime associated with $\mathrm{SF} f$, and $\lambda$ is the transmission rate of all nodes.

The DER is usually computed for each SF in which all nodes using the SF are considered interfering with each other. A collision may occur between two simultaneous LoRa frames in the same frequency and using the same SF. In reality, colliding transmissions may still be received due to the capture effect. The frame with the highest power can be decoded if the received power at the gateway is at least $6 \mathrm{~dB}$ more than the other frame (i.e. 4 times stronger) [1]. In this work, we use this more accurate channel representation with physical capture, and the resulting DER is increased.

The capture effect can be modeled as follows, considering the average received signal power at the gateway. Let $C_{i j}$ be a parameter indicating if the LoRa frames of nodes $i$ and $j$ using the same SF $f$ can collide. By definition:

$$
C_{i j}^{f}= \begin{cases}1 & \text { if } P_{r x}^{i}-P_{r x}^{j} \leq 6, \\ 0 & \text { otherwise }\end{cases}
$$

In an actual LoRaWAN deployment, each transmitted message should be received correctly by at least one gateway. In case there are several gateways, two colliding node transmissions $i$ and $j$ in the same SF are lost if the power difference is less than $6 \mathrm{~dB}$ at all the gateways. Otherwise, there exists at least one gateway that is capable of decoding $i$ 's frame while $j$ is transmitting too.

Considering a node $i \in \mathcal{I}$ using SF $f$, the number of nodes potentially interfering on SF $f$ with $i$ is thus $\sum_{j \in \mathcal{I}, j \neq i} C_{i j}^{f}$. By definition, this number is always lower or equal to the total number of nodes using $\mathrm{SF} f$.

\section{Imperfect SF orthogonality}

Two nodes using different SFs can transmit their data simultaneously, as long as none is received with a power significantly higher [8]. If the transmitting nodes use different $\mathrm{SF}$, then each packet can be demodulated if the difference between the received power is greater than the SINR (Signalto-Interference-Plus-Noise-Ratio) threshold of each SF (cf Table I). For instance, a transmission at SF 7 is received as long as another transmission at SF 8 is no more than $16 \mathrm{~dB}$ 
TABLE I: SINR thresholds for co-SF interference

\begin{tabular}{r|r|r|r|r|r|r}
$\begin{array}{r}\text { interferer } \\
\text { desired }\end{array}$ & 7 & 8 & 9 & 10 & 11 & 12 \\
\hline 7 & 6 & -16 & -18 & -19 & -19 & -20 \\
8 & -24 & 6 & -20 & -22 & -22 & -22 \\
9 & -27 & -27 & 6 & -23 & -25 & -25 \\
10 & -30 & -30 & -30 & 6 & -26 & -28 \\
11 & -33 & -33 & -33 & -33 & 6 & -29 \\
12 & -36 & -36 & -36 & -36 & -36 & 6
\end{tabular}

stronger (i.e. 40 times stronger). We define parameter $I_{i j}^{f f^{\prime}}$ for inter-SF potential collisions between SF $f$ and $f^{\prime}$.

$$
I_{i j}^{f f^{\prime}}= \begin{cases}1 & \text { if } P_{r x}^{i}-P_{r x}^{j} \leq S I N R_{f f^{\prime}} \\ 0 & \text { otherwise }\end{cases}
$$

where $S I N R_{f f^{\prime}}$ is the value of the required SINR when the transmitting node $i$ use SF $f$ and the other simultaneous transmission is done using SF $f^{\prime} \neq f$ (cf. Table I). In the multiple gateways case, $I_{i j}^{f f^{\prime}}=1$ if all the gateways receive $P_{r x}^{i}-P_{r x}^{j} \leq S I N R_{f f^{\prime}}$. For a node $i \in \mathcal{I}$ using SF $f$, the number of nodes generating potential inter-SF collisions with $i$ is $\sum_{f^{\prime} \neq f} \sum_{j \in \mathcal{I}, j \neq i} I_{i j}^{f f^{\prime}}$.

\section{Success probability}

Given the total number of potential interferers $N_{i}=$ $\sum_{j \neq i} C_{i j}^{f}+\sum_{f^{\prime} \neq f} \sum_{j \neq i} I_{i j}^{f f^{\prime}}$ of node $i \in \mathcal{I}$ using SF $f$, we have to ensure that none of these interferers starts a transmission within $2 T^{f}$ to avoid overlap. The probability of successful transmission for node $i$ thus equals

$$
\operatorname{Pr}(i)=e^{\left(-2 T^{f} \lambda N_{i}\right)}
$$

where $\lambda$ is the traffic intensity and $T^{f}$ is the transmission air time at SF $f$ used by node $i$ (cf. Table II).

Given a LoRa network and a set of nodes $\mathcal{I}$ deployed on the covered area, we seek to optimally allocate the SF and maximize the number of nodes supported by the network so that the transmission success probability of each served node is greater than a threshold $\gamma$.

\section{Optimal SF ALLOCATION}

Let $y_{i}^{f}$ be a binary variable stating that spreading factor $f$ has been assigned to sensor $i \in \mathcal{I}$, with $f \in\{7, \ldots, 12\}$. We say that a node $i \in \mathcal{I}$ is served if $\sum_{f \in\{7, \ldots, 12\}} y_{i}^{f}=1$. The model seeks to maximize the number of served nodes in the network, while allocating the spreading factors to the served nodes in order to maximize their transmission quality.

The optimal framework for SF allocation is given by the following integer linear program (ILP) :

$$
\begin{array}{rc}
\max \sum_{i \in \mathcal{I}} \sum_{f \in\{7, \ldots, 12\}} \omega_{i} y_{i}^{f} & \\
\sum_{f \in\{7, \ldots, 12\}} y_{i}^{f} \leq 1 & \forall i \in \mathcal{I} \\
H \geq \beta y_{i}^{f} \quad \forall i \in \mathcal{I}, f \in\{7, \ldots, 12\}
\end{array}
$$

Objective (5) maximizes the number of served nodes in the network by maximizing $\sum_{i} \sum_{f} y_{i}^{f}$. We associate a weight parameter $\omega_{i}$ to the selection of each node $i$ in order to give a priority in the SF allocation. If a node can be served, then we want to allocate it with the smallest SF possible while meeting the global requirements in terms of transmission probability. We choose a weight that decreases with the spreading factor in order to encourage small SF for the selected nodes and avoid large SF near the gateway. Indeed, without $\omega_{i}$, if a node is served and cannot be allocated with its smallest possible SF, then the model has no indication to encourage the SF directly higher and can allocate an arbitrary large SF no matter what is the distance to the gateways. So we select for instance $\omega_{i}=(1-H)$ since probability $H$ increases with the SF. At most one SF $f$ can be assigned to a node $i \in \mathcal{I}$ (Constraints (6)) if it meets the signal strength reception condition for at least one LoRa gateway (with probability H) (Constraints (7)).

Then, we want the transmission success probability to be greater than $\gamma$ for all the nodes. Since we do not know what spreading factor the nodes will use, we define the following set of constraints

$$
\begin{aligned}
e^{-2 \lambda} \sum_{f \in\{7, \ldots, 12\}} T^{f} y_{i}^{f}\left(1+\sum_{j \neq i} C_{i j}^{f} y_{j}^{f}+\sum_{f^{\prime} \neq f} \sum_{j \neq i} I_{i j}^{f f^{\prime}} y_{j}^{f^{\prime}}\right) & \geq \gamma \\
\forall i & \in \mathcal{I}
\end{aligned}
$$

These constraints are non linear because of the expression of $\operatorname{Pr}(i)$. We linearize them using the log function. Constraints (8) are thus equivalent to:

$$
\sum_{f} T^{f} y_{i}^{f}\left(1+\sum_{j \neq i} C_{i j}^{f} y_{j}^{f}+\sum_{f^{\prime} \neq f} \sum_{j \neq i} I_{i j}^{f f^{\prime}} y_{j}^{f^{\prime}}\right) \leq-\frac{\log (\gamma)}{2 \lambda}
$$

This equation is still non linear. By equation (6), we know that $\sum_{f} y_{i}^{f}=0$ or 1 for exactly one $f \in\{7, \ldots, 12\}$. So we want to ensure

$$
T^{f}\left(1+\sum_{j \neq i} C_{i j}^{f} y_{j}^{f}+\sum_{f^{\prime} \neq f} \sum_{j \neq i} I_{i j}^{f f^{\prime}} y_{j}^{f^{\prime}}\right) \leq-\frac{\log (\gamma)}{2 \lambda}
$$

for $y_{i}^{f}=1$, and have no constraint on the probability reception if $y_{i}^{f}=0$. We decompose the set of constraints for each $i \in \mathcal{I}$ and $f \in\{7, \ldots, 12\}$ :

$$
\begin{aligned}
& T^{f}\left(1+\sum_{j \neq i} C_{i j}^{f} y_{j}^{f}+\sum_{f^{\prime} \neq f} \sum_{j \neq i} I_{i j}^{f f^{\prime}} y_{j}^{f^{\prime}}\right) \\
& \leq-\frac{\log (\gamma)}{2 \lambda}+M\left(1-y_{i}^{f}\right), \quad \forall i \in \mathcal{I}, f \in\{7, \ldots, 12\}
\end{aligned}
$$

where $M$ is a sufficiently large constant. When $y_{i}^{f}=0$ (the $\mathrm{SF}$ is not selected), this constant makes the left-hand side bounded by a very large value, making it unconstrained. The constraint associated with node $i$ for a non allocated SF $f$ has therefore no impact on the SF allocation of all the nodes and the success probabilities. When $y_{i}^{f}=1(f$ is allocated to $i$ ), then $M$ disappears in the associated constraint (9) and we get the equivalence of constraint (8) as requested to ensure the success probability of node $i$.

Our optimization problem is solved considering the integer linear program (5) (6) (7) (9). 

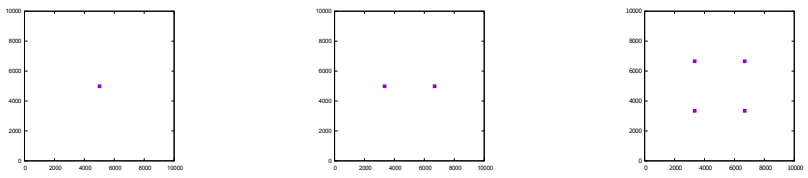

Fig. 1: LoRaWAN with regular gateway location.

TABLE II: Numerical values for a $\lambda=59$ byte packet at $B W=125 \mathrm{kHz}$

\begin{tabular}{c|c|c} 
SF $f$ & Time on air $T^{f}(\mathrm{~ms})$ & Required SNR $q_{f}(\mathrm{~dB})$ \\
\hline 7 & 102 & -6 \\
8 & 184 & -9 \\
9 & 328 & -12 \\
10 & 616 & -15 \\
11 & 1315 & -17.5 \\
12 & 2466 & -20
\end{tabular}

\section{Results}

\section{A. Scenario}

We consider an $10 \mathrm{~km} \times 10 \mathrm{~km}$ square area with one, two, or four gateways regularly positioned, cf. Figure 1 . We randomly deploy $N$ nodes in this area, $N$ varying from 50 to 1000 nodes depending on the number of gateways and the probability threshold $\gamma$. For each value of $N$, we generates 10 random test instances.

Regardless of medium access contention, it is reasonable to set the SF of each node so that it meets a given target packet delivery ratio. In our model, we allow SF $f$ to be allocated to a node if the probability of success $H$ is above $\beta=66 \%$. To ensure this requirement, we use the SNR threshold values defined in Table II. We thus obtain topologies in which the distribution of nodes within each SF annulus is $[33 \% ; 15 \% ; 21 \% ; 22 \% ; 8 \% ; 1 \%]$.

We assume that all nodes have the same traffic intensity, in which case it is relevant to express the cell capacity in terms of number of nodes. This assumption is the most realistic, since traffic intensity $\lambda$ depends on the application traffic generation pattern. We consider a network with a single application, in which case all nodes produce the same traffic. In LoRaWAN, nodes have to limit their occupation of each frequency band to $1 \%$ of time. We set $\lambda$ to the maximum intensity for any node in the system, which is determined by the "slowest nodes" (at high SF) at their authorized duty cycle limit, and using $59 \mathrm{~B}$ frames, the maximum size at this rate. It corresponds to $2.47 \mathrm{~s}$ of airtime, thus $\lambda=\frac{1}{747 \mathrm{~s}}$. Airtime values for all SF are presented in Table II.

The model is implemented in Java and solved using IBM CPLEX solver 12.8 on an Intel Core i7-5500U CPU, 2.40 $\mathrm{GHz}, 32 \mathrm{~Gb}$ RAM computer under Linux Fedora operating system. The resolution time limit of CPLEX has been set to 1 h.

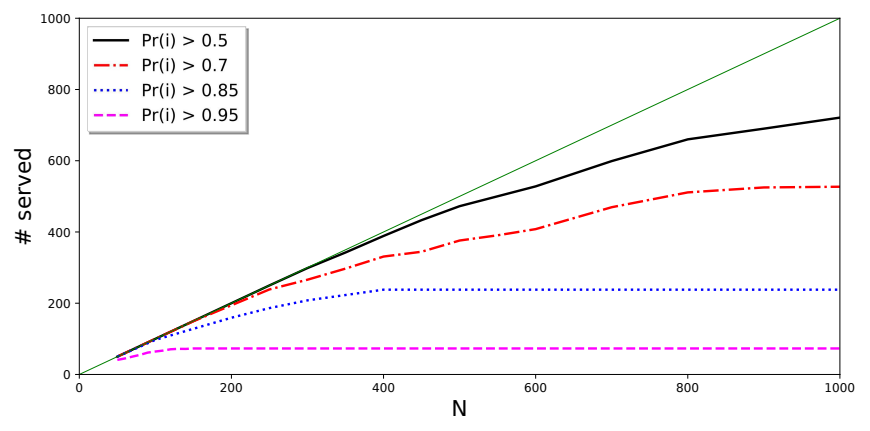

(a) Single cell

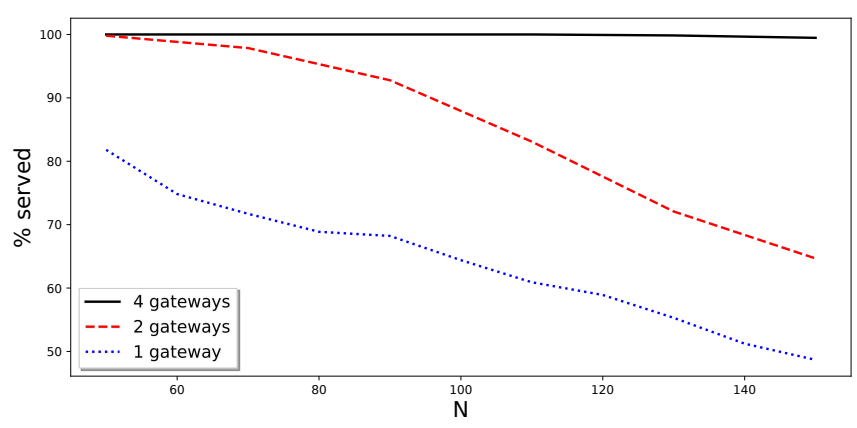

(b) Multiple gateway gain, $\gamma=95 \%$

Fig. 2: Capacity analysis.

\section{B. Network performances}

We first evaluate a LoRaWAN cell capacity in terms of number of served nodes. Figure $2 \mathrm{a}$ depicts the number of served nodes in function of $N$, the number of deployed nodes in the cell of a LoRa gateway. All nodes can be served for small-sized networks (following the green line $y=x$ in the figure), except for very high required success probability. The number of served nodes increases with $N$ until it reaches a maximum value corresponding to the maximum cell capacity. For instance, 73 nodes can be served at most in a cell with $\operatorname{Pr}(i) \geq \gamma=95 \%$ (purple dashed line), and we reach this value when $N \geq 150$. Capacity limit equals 238 nodes for $\gamma=85 \%$ and $N \geq 400,527$ nodes for $\gamma=70 \%$ and $N \geq 900$, and more than 720 nodes for $\gamma=50 \%$ since the limit has not been reached for $N=1000$.

Increasing the number of gateways in the network provides a capacity gain quantified in Figure $2 b$ in the case where all nodes cannot be served. The gain is at least $20 \%$ for $\gamma=95 \%$ when we double the gateways in the area, given the same set of deployed nodes. And for $\gamma=50 \%$, even with 2 gateways, $100 \%$ of nodes can be served for all values of $N \in[50,1000]$, which gives already a gain of $30 \%$ for $N=1000$ compared to the single cell capacity.

In an effective LoRaWAN deployment, all transmitted messages should be received by the NetServer, the network server connected to all the LoRa gateways. This means that each transmitted message should be received correctly by at least one gateway. The only case where the NetServer does not 


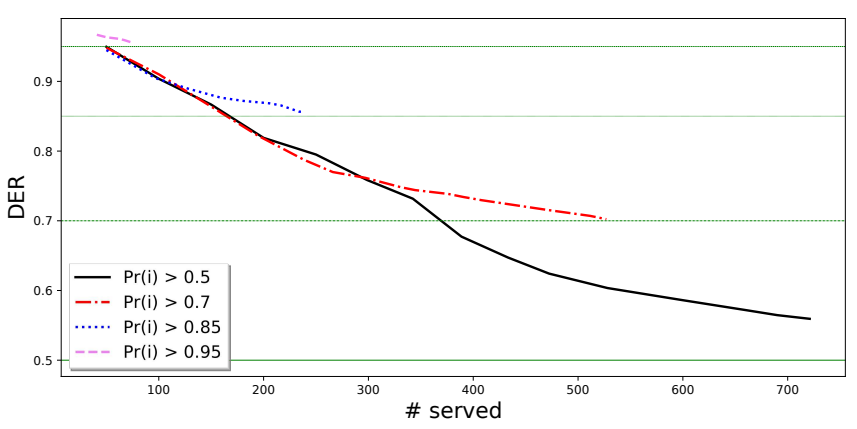

(a) Single cell

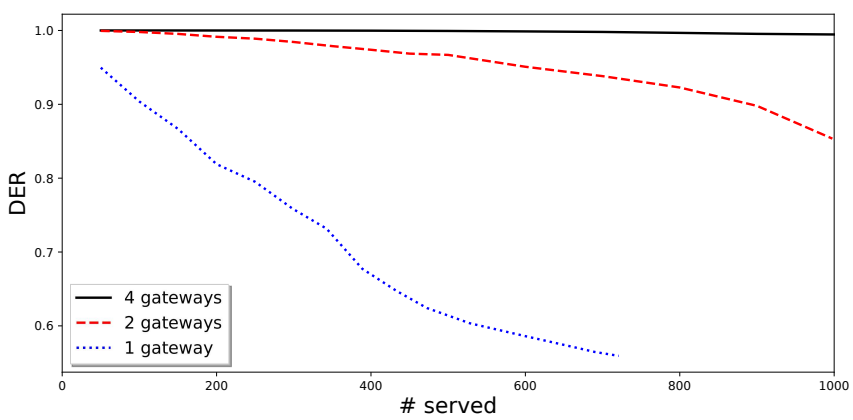

(b) Multiple gateway gain, $\gamma=50 \%$

Fig. 3: DER analysis.

receive the message is the one in which all the gateways fail to receive it correctly. This case happens to gateway $g \in \mathcal{G}$ with probability $\left(1-\mathrm{DER}_{g}\right)$. The data extraction rate at the NetServer is thus the following:

$$
\mathrm{DER}_{\text {NetServer }}=1-\prod_{g \in \mathcal{G}}\left(1-\mathrm{DER}_{g}\right)
$$

We evaluate the mean data extraction rate at the network server for different values of $\gamma$ and number of gateways. The DER decreases when the number of served nodes increases while converging to $\gamma$ when the network capacity reaches its limit (Figure 3a).

Figure $3 \mathrm{~b}$ reports the number of served nodes and the average data extraction rate for different number of deployed gateways when the minimum required success probability is set to $50 \%$. With one gateway, the DER decreases linearly when the number of served nodes increases, going from 0.95 for 40 served nodes to 0.56 for 720 nodes. Only doubling the number of gateways allows to increase the DER to 0.93 for 720 nodes. Then, with 4 gateways, the DER always stays greater than 0.99 until 1000 served nodes.

\section{SF allocation}

Our model evaluates the capacity of a LoRa network while optimizing the allocation of the spreading factors to the served nodes balancing attenuation and collisions. We now evaluate this allocation by comparing it to the distance-based $\mathrm{SF}$ allocation. In this case, the allocation depends on the distance to the gateways and the propagation model. The smallest $\mathrm{SF}$ achieving that $H \geq \beta$ is allocated to the node: $\mathrm{SF}_{i}=\min \mathrm{SF}$ such that $H \geq \beta$. In other words, the lowest possible $\mathrm{SF}$ is allocated to each node. In case of multiple gateways, the selected SF is the minimum SF among all the reachable gateways.

For sparse cells, our SF allocation (optSF) provides the same capacity as the distance-base SF allocation (minSF). However for dense cells, we observe that our policy gives better results in terms of number of served nodes (Figure 5). These results highlights the fact that the SF boundaries should be carefully optimized. Figure 4 depicts the optimal SF allocation for $N=400$ and $\gamma=50 \%$. By looking at the allocated SF, one can remark that around the SF boundaries, some nodes may receive SF $f+1$ while being closer to nodes at SF $f$. Our model allocates the best SF optimizing both attenuation and collisions.

\section{Impact of physical capture}

We first evaluate only the impact of considering physical capture on the LoRaWAN capacity. We compare two policies:

1) $\mathrm{SF}$ allocation without capture effect and with perfect $\mathrm{SF}$ orthogonality. All nodes in the same SF are interfering with each other. In constraints (9), all $C_{i j}^{f}$ are set to 1 , and all $I_{i j}^{f f^{\prime}}$ to 0 .

2) SF allocation with physical capture and with perfect SF orthogonality. In constraints (9), we only consider $C_{i j}^{f}$ parameters as defined in Section II, and all $I_{i j}^{f f^{\prime}}$ are still 0 .

As expected, the capacity increases when considering the capture effect arising at the gateways. We quantify this gain in Figure 6 for $\gamma=95 \%$. The number of served nodes for each policy is normalized against case 2 (called "Capture"). We see that without physical capture ("No capture"), the capacity loss is around $8 \%$ for all values of $N$. And we have seen that at the capacity limit, 6 nodes cannot be served without considering physical capture.

\section{E. Impact of imperfect SF orthogonality}

We now include the consideration of imperfect SF orthogonality in the capacity of the network. We compare the results obtained by our ILP (with $C_{i j}^{f}$ and $I_{i j}^{f f^{\prime}}$ computed as defined in Section II), with the two policies presented in the previous section.

The imperfect SF orthogonality has an effect mainly between nodes located near the gateways (using SF7) and those far away (using SF11 or 12). This affects the total number of served nodes in the network when the nodes too close to the gateway cannot be served (see for instance Figure 4a). The capacity with imperfect SF orthogonality ("Capture + co-SF" on Figure 6) is lower than the one without (labeled "Capture", case 2 of the previous section) when the capacity limit has not been reached. However, considering imperfect SF orthogonality does not impact the network capacity since the same number of served nodes can be achieved in both cases (Figure 6). 


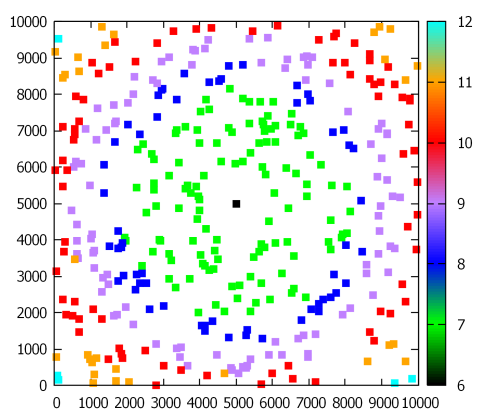

(a) 1 gateway, 387 of 400 nodes served

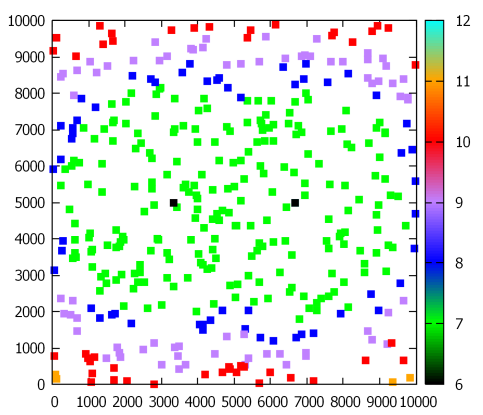

(b) 2 gateways, all 400 nodes served

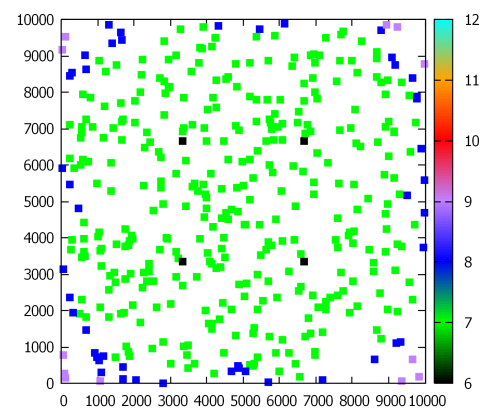

(c) 4 gateways, all 400 nodes served

Fig. 4: Optimal SF allocation for a network of $N=400$ and $\gamma=50 \%$.

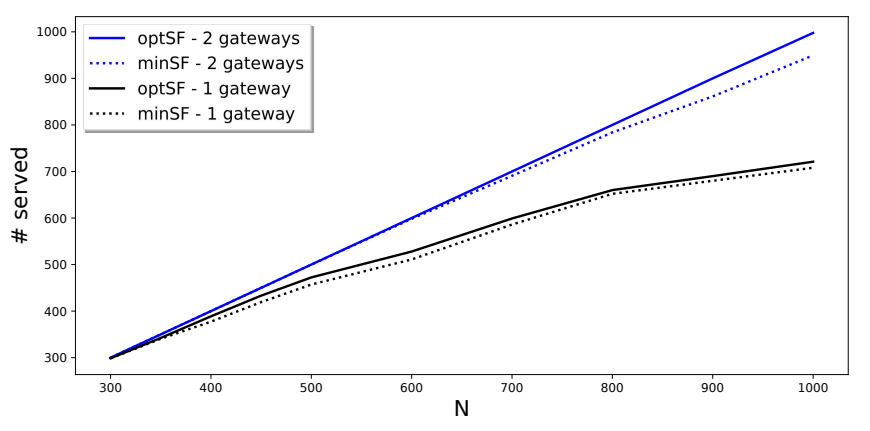

Fig. 5: Comparison between SF allocation policies.

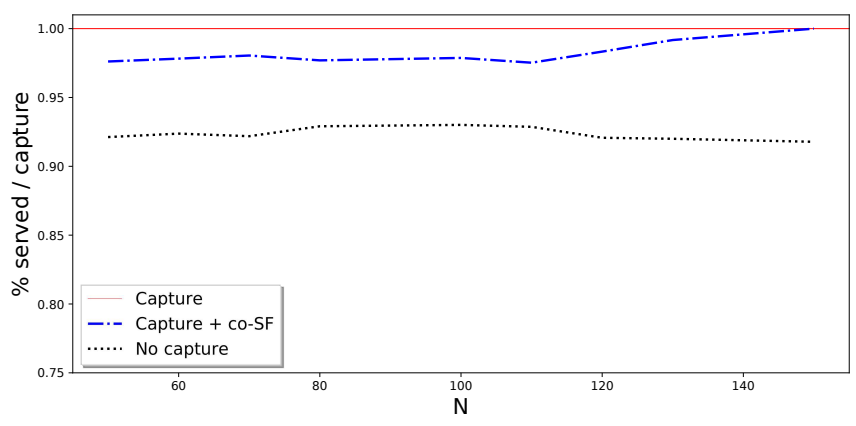

Fig. 6: Effects of physical capture and SF orthogonality.

\section{Conclusion}

In this paper, we present an optimal framework for the SF allocation problem in order to maximize the number of served nodes in a LoRaWAN network while ensuring a transmission success probability to the nodes. We develop and optimally solve an integer linear program to tackle the problem. The originality of our work lies on the focus of our approach which is the maximization of the network capacity expressed as the number of served nodes, taking into account the physical capture at the LoRaWAN gateways and imperfect SF orthogonality. The simulation results show the effectiveness of our strategy both in terms of deployment and computation cost. Following this work, we would like to validate this allocation by simulation and experimentally to quantify the benefits of proper SF allocation in terms on packet delivery ratio in a realistic environment. Another improvement is to optimally determine the SF boundaries in function of the maximum transmission radius and isolated frame reception probability. This would be of great interest to evaluate the trade-off between maximum transmission range (attenuation) and the impact of imperfect SF orthogonality.

\section{REFERENCES}

[1] O. Georgiou and U. Raza, "Low power wide area network analysis: Can lora scale?" IEEE Wireless Communications Letters, vol. 6, no. 2, pp. 162-165, April 2017.

[2] J. de Carvalho Silva, J. J. P. C. Rodrigues, A. M. Alberti, P. Solic, and A. L. L. Aquino, "Lorawan - a low power wan protocol for internet of things: A review and opportunities," in 2017 2nd International Multidisciplinary Conference on Computer and Energy Science (SpliTech), July 2017.

[3] N. Varsier and J. Schwoerer, "Capacity limits of lorawan technology for smart metering applications," in 2017 IEEE International Conference on Communications (ICC), May 2017.

[4] M. C. Bor, U. Roedig, T. Voigt, and J. M. Alonso, "Do lora lowpower wide-area networks scale?" in Proceedings of the 19th ACM 
International Conference on Modeling, Analysis and Simulation of Wireless and Mobile Systems, ser. MSWiM, 2016, pp. 59-67.

[5] B. O. A. Yousuf, E. Rochester and M. Ghaderi, "Throughput, coverage and scalability of lora lpwan for internet of things," in IEEE/ACM International Symposium on Quality of Service (IWQoS), June 2018.

[6] A. Duda and M. Heusse, "Spatial issues in modeling lorawan capacity," in 22nd ACM International Conference on Modeling, Analysis and Simulation of Wireless and Mobile Systems (MSWIM), 112019.

[7] D. Bankov, E. Khorov, and A. Lyakhov, "Mathematical model of lorawan channel access with capture effect," in 2017 IEEE 28th Annual International Symposium on Personal, Indoor, and Mobile Radio Communications (PIMRC), Oct 2017.

[8] C. Goursaud and J. M. Gorce, "Dedicated networks for iot: Phy / mac state of the art and challenges," EAI Endorsed Transactions on Internet of Things, vol. 1, no. 1, 102015.

[9] A. Waret, M. Kaneko, A. Guitton, and N. El Rachkidy, "LoRa Throughput Analysis with Imperfect Spreading Factor Orthogonality," IEEE Wireless Communications Letters, vol. 8, no. 2, pp. 408-411, Apr. 2018.

[10] A. Mahmood, E. Sisinni, L. Guntupalli, R. Rondon, S. A. Hassan, and M. Gidlund, "Scalability analysis of a lora network under imperfect orthogonality," IEEE Transactions on Industrial Informatics, 2018.

[11] M. Cesana, A. Redondi, and J. Ortìn, "A framework for planning lorawan networks," in IEEE International Symposium on Personal Indoor and Mobile Radio Communications (PIMRC), September 2018.

[12] D. Zorbas, G. Z. Papadopoulos, P. Maillé, N. Montavont, and C. Douligeris, "Improving lora network capacity using multiple spreading factor configurations," in 25th International Conference on Telecommunications, ICT, 2018, pp. 516-520.

[13] D. Zorbas, P. Maillé, B. O'flynn, and C. Douligeris, "Fast and reliable lora-based data transmissions," in 24th Symposium on Computers and Communications (ISCC), 062019.

[14] J. Lim and Y. Han, "Spreading factor allocation for massive connectivity in lora systems," IEEE Communications Letters, vol. 22, no. 4, pp. 800803, April 2018. 